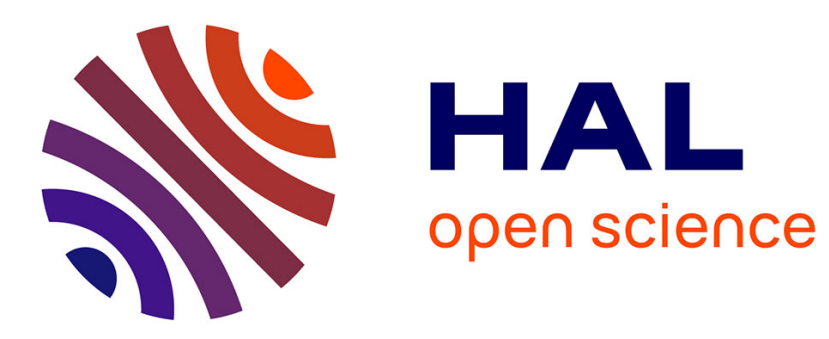

\title{
Stability of solid phases in the dipolar hard sphere system
}

Dominique Levesque, Jean-Jacques Weis

\section{To cite this version:}

Dominique Levesque, Jean-Jacques Weis. Stability of solid phases in the dipolar hard sphere system. Molecular Physics, 2012, 109 (23-24), pp.2747-2756. 10.1080/00268976.2011.610368 . hal-00771582

\section{HAL Id: hal-00771582 \\ https://hal.science/hal-00771582}

Submitted on 9 Jan 2013

HAL is a multi-disciplinary open access archive for the deposit and dissemination of scientific research documents, whether they are published or not. The documents may come from teaching and research institutions in France or abroad, or from public or private research centers.
L'archive ouverte pluridisciplinaire HAL, est destinée au dépôt et à la diffusion de documents scientifiques de niveau recherche, publiés ou non, émanant des établissements d'enseignement et de recherche français ou étrangers, des laboratoires publics ou privés. 


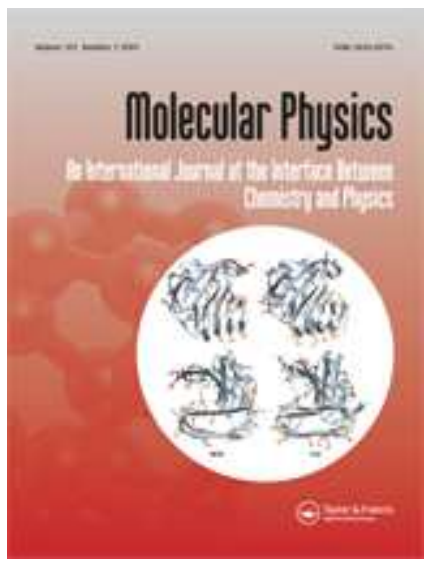

\section{Stability of solid phases in the dipolar hard sphere system}

\begin{tabular}{|r|l|}
\hline Journal: & Molecular Physics \\
\hline Manuscript ID: & TMPH-2011-0166.R1 \\
\hline Manuscript Type: & Special Issue in honour of Luciano Reatto \\
\hline Date Submitted by the & 07-Jul-2011 \\
\hline Complete List of Authors: & $\begin{array}{l}\text { Levesque, Dominique; Université de Paris-Sud XI, Laboratoire de } \\
\text { Physique Théorique } \\
\text { Weis, Jean-Jacques; Université Paris-Sud XI, Laboratoire de } \\
\text { Physique Théorique }\end{array}$ \\
\hline Keywords: & $\begin{array}{l}\text { solid phases, dipolar hard spheres, Monte Carlo simulation, } \\
\text { nonequilibrium work method }\end{array}$ \\
\hline $\begin{array}{l}\text { Note: The following files were submitted by the author for peer review, but cannot be converted } \\
\text { to PDF. You must view these files (e.g. movies) online. }\end{array}$ \\
\hline $\begin{array}{l}\text { dipsol_final.tex } \\
\text { tMPH2e.cls }\end{array}$ \\
\hline
\end{tabular}

\section{SCHOLARONE" \\ Manuscripts}




\title{
RESEARCH ARTICLE
}

\section{Stability of solid phases in the dipolar hard sphere system}

\author{
D. Levesque* and J. -J. Weis ${ }^{\dagger}$ \\ Université de Paris-Sud, Laboratoire de Physique Théorique, UMR8627, \\ Bâtiment 210, 91405 Orsay Cedex, France \\ (Received 00 Month 200x; final version received 00 Month 200x)
}

\begin{abstract}
Free energy differences between solid phases of dipolar hard spheres are estimated by Monte Carlo simulation using a nonequilibrium work method. These calculations allow to determine which of the considered phases has the minimum free energy. The phase diagram which we obtain is confirmed by simulations in the isothermal-isobaric ensemble over a wide region of the density and temperature domain.
\end{abstract}

\section{Keywords: solid phases; dipolar hard spheres; Monte-Carlo simulations; nonequilibrium} work method

\section{Introduction}

We are very pleased to contribute to this special issue in honour of Professor Luciano Reatto and, on this occasion, to remind us of our fruitful collaboration.

The dipolar hard sphere (DHS) system presents a variety of fluid and solid states which have been actively studied in the literature [1]. The low density states, at small dipole moments are similar to those in simple monatomic fluids. At moderate densities and large dipole moments, in the fluid state, the DHS associate into linear chains of variable lengths [2-4]. Much endeavour has been devoted as to whether there could be liquid-vapour type coexistence between the low density fluid phase and the one of linear aggregates without, though, being able to provide an unambiguous conclusion about the existence of such a coexistence and the location of its critical point [5-7]. At densities typical of the liquid state and large dipole moments simulation results established the existence of a polarized phase for finite size systems with periodic boundary conditions [8-10]. The existence of such a phase in the thermodynamic limit has, however, been questioned on the basis of theoretical arguments [11, 12]. Nonetheless, the dense fluid phases, polarized or not, coexist with solid phases whose symmetry has, to a large extent, still to be established. The aim of the present work is to contribute to the determination of the diagram of the solid phases and to estimate their stability as a function of density and dipole moment, i.e. temperature in the present case.

Available studies at zero or finite temperatures demonstrated the existence of a variety of solid phases for dipolar particles of spherical shape [13-17]. At low dipole moments there will be no polarization and the stable lattices should, a priori, be similar to those found for spherically symmetric particles interacting by a van der Waals type pair potential. These lattices are of a body-centred cubic (bcc) type

\footnotetext{
*Email:dominique.levesque@th.u-psud.fr

$\dagger^{\dagger}$ Corresponding author.Email: jean-jacques.weis@th.u-psud.fr 
at high temperature and face-centred cubic (fcc) or hexagonal close-packed (hcp) at low temperature [18]. For sufficiently high dipole moments such that the solid phase is polarized, the unit cell of the lattice can be modified. If, for instance, there is a polarization along the z-axis the cell edge of the bcc lattice can decrease in this direction and the bcc lattice transforms into a body-centred tetragonal (bct) lattice. If, in addition, the lattice parameters change along the $\mathrm{x}$ and $\mathrm{y}$ axes but the unit cell remains tetragonal the bcc lattice turns into a base-centred orthorhombic (bco) lattice. Similarly, fcc or hcp lattices can modify under the influence of polarization.

The number of possible lattices in the solid phase, taking into account the dipolar interaction and excluded volume effects, can obviously be quite large. In the present study only tetragonal lattice cells, bcc, fcc, bct, bco and also hcp will be considered. The relative stabilities of the corresponding phases will be established by Monte Carlo (MC) simulation.

A frequently used method to estimate free energy differences between phases is thermodynamic integration [19]. This method assumes that the free energy difference can be calculated along a sequence of equilibrium states connecting a reference state of known free energy to the equilibrium state of the solid phase under consideration. This procedure requires that no phase transition is crossed when going from the initial reference state to the final equilibrium state. The possibility of the existence of first order phase transitions between solid phases as well as the absence of a convenient reference system for systems with rotational degrees of freedom determined us to discard this method in the present study.

Monte Carlo simulations in the isobaric ensemble [19] are in principle suitable for determining solid phase diagrams. With an adequate choice of particle number, MC sampling of the size and the shape of the simulation volume possibly can drive the system to evolve e.g., from a bcc to a fcc phase or conversely, depending on their relative stability at the density and temperature considered. The principal limitation of this method is that despite a favourable free energy difference, MC sampling over an accessible amount of computation time may succeed the system to evolve only from an unstable phase to a mechanically more stable phase but not to the phase of minimum free energy. Due to this limitation we supplemented the simulations in the isobaric ensemble with an estimate of the free energy difference of the solid phases based on the Jarzynsky equality [20], also referred to as the nonequilibrium work (NEW) method in the literature. The latter allows to calculate the difference between two equilibrium states from the work which is spent when going from one state to the other. This calculation, contrary to those based on thermodynamic integration or determination of the mean work, does not require reversible transformations. However, for given temperature (or dipole moment) and density, the Jarzynski equality does not allow to access the minimum free energy of the solid phase. Indeed, the free energy difference is evaluated between a given initial equilibrium state and final states which are specified transformations of this initial state. The calculated free energies allow only to estimate the relative stability of the chosen final states. When these final states are similar to the solid phases spontaneously obtained in the isobaric ensemble simulations, it is possible to distinguish which among them is the state of lowest free energy.

The article is organized as follows. Section 2 gives details of the simulation methods and the use of the Jarzynski equality. Section 3 presents the simulation results in the isobaric ensemble and for the free energy differences. In Section 4 a tentative solid phase diagram of the DHS system is given in the density range $\rho^{*}=\rho \sigma^{3}$ $=1.06-1.26$ and dipole moments $\mu^{*}=\mu / \sqrt{k_{B} T \sigma^{3}}=1,2$ and 3 where $\sigma$ and $\mu$ are the diameter and dipole moment of the spheres and $T$ the temperature. A 
summary of the main results is given in the conclusion.

\section{Simulation method and free energy calculation.}

The simulations in the isobaric ensemble are performed at fixed temperature and pressure, with the standard sampling algorithm alternating trial displacements of the particles and trial variations of the size and shape of the volume containing the system of $N$ hard spheres with periodic boundary conditions. Shape variations are such that the simulation cell remains tetragonal. Having fixed $N$, the pressure and dipole moment and chosen an initial lattice and volume, the MC sampling will drive this state to one with lower free energy. From the analysis of the pair distribution functions $g(r)$ and the average dimensions of the simulation volume in the final equilibrium state one can determine the symmetry of the lattice.

Isobaric-isothermal (NpT) MC were performed for dipole moments $\mu^{*}=1,2$ and 3 by applying a uniform pressure $p$ to an initial system having bcc, bct, fcc or hcp structure. By varying $p / k_{B} T$ from 2 to 35 a density range $\rho^{*}=1.06-1.27$ is covered. The number of particles was 1024, 1280, 864 and 1440 for the bcc, bct, fcc and hcp lattices, respectively. For each state the initial orientations of the dipole moments were chosen to be random and the total energy of the system was calculated by means of an Ewald sum [21]. An attempted volume change is achieved by varying the three box dimensions separately according to $L_{\alpha}^{\prime}=L_{\alpha}\left(1+\Delta_{\alpha}\left(0.5-\lambda_{\alpha}\right)\right)$ where the $\lambda_{\alpha}(\alpha=x, y, z)$ are random numbers in the interval $[0,1]$, maintaining the simulation cell orthogonal. The values of $\Delta_{\alpha}$ were adjusted to have an acceptance ratio for the volume changes of about 0.3-0.4.

Ideally one would expect that whatever the initial lattice structure of the system, the MC procedure would drive it to the configuration of lowest free energy (within the constraint of an orthogonal cell). It turned out that for most states considered the initial compact lattices, hcp and fcc, remained stable and did not transform to the lattice structure identified by the NEW method described below as being of lowest free energy. As will be stated below the transformation of an initial hcp structure to e. g. a bcc or bco structure requires relative displacements of alternate basal planes but such moves had not been implemented in the MC sampling. Starting from an initial fcc lattice, which remains quite stable and becomes polarized for $\mu^{*}>1$, the dipole arrangement most oftenly obtained consists of domains of lattice planes in which the dipole moments all point in the same direction (on average). The direction is tilted by $45^{\circ}$ with respect to the average total polarization vector (e.g. along $x$ axis). While the tilt angle is the same for all domains, the average orientations of the dipole moment projections onto the $y z$ plane (perpendicular to the polarization) are located along the plane diagonals, and vary from one domain to the other by a rotation of $90^{\circ}$. Figure 1 gives a view of this dipole arrangement projected on the $x y$ plane. Such a structure had already been evidenced in previous canonical (NVT) MC simulations [13]. On the other hand, when the initial state was chosen to be a bcc or bct lattice the system generally evolved to a state compatible with that of lowest free energy as obtained by the NEW method. Examples will be given below.

The Jarzynski equality [20]

$$
<e^{-W / k_{B} T}>=e^{-\left(F_{F}-F_{I}\right) / k_{B} T}=e^{-\Delta F / k_{B} T}
$$

enables to calculate the free energy difference $\Delta F$ between equilibrium states $I$ and $F$ of a system by evaluating the average of $e^{-W / k_{B} T}$ along a set of paths, i.e. a set 
of configurations relating the states $I$ and $F$. $W$ is the work done on the system in the transition $I$ to $F$ along one such path. As repeatedly proven and discussed in the literature, paths from $I$ to $F$ can be configurations obtained from hamiltonian dynamics [20, 22, 23] or from a Markov process satisfying detailed balance [24, 25]. It is the last category of paths which has been used in the present work. More specifically, the initial state of a path is a system of $N$ hard spheres at density $\rho_{I}^{*}=\rho_{I} \sigma^{3}$ and dipole moment $\mu^{*}$ located on a bcc lattice of volume $V$ with periodic boundary conditions defined by the three vectors $\mathbf{a}, \mathbf{b}$ and $\mathbf{c}$ corresponding to the columns of the matrix $h_{\alpha \beta}^{I}$ with $\operatorname{det}\left(h_{\alpha \beta}^{I}\right)=V$. The system in this initial state is transformed into a state of same volume $V$ specified by the matrix $h_{\alpha \beta}^{F}$. The matrix $h_{\alpha \beta}^{F}$ is such that the shape of the volume $V$ corresponds to $N$ hard spheres located on a fcc, bct, bco or hcp lattice at density $\rho_{F}^{*}=\rho_{I}^{*}$. This transformation is achieved by a succession of matrices $h_{\alpha \beta}^{i}(i=0,1, \ldots, n)$ with $h_{\alpha \beta}^{0}=h_{\alpha \beta}^{I}, h_{\alpha \beta}^{n}=h_{\alpha \beta}^{F}$ and $h_{\alpha \beta}^{i}=h_{\alpha \beta}^{i-1}+\delta h_{\alpha \beta}^{i}$. The small random variations $\delta h_{\alpha \beta}^{i}$ are given by

$$
\delta h_{\alpha \beta}^{i}=\left(\lambda_{i}-\lambda_{i-1}\right)\left(h_{\alpha \beta}^{F}-h_{\alpha \beta}^{I}\right)
$$

where the variables $\lambda_{i}(0<i<n)$ are a sequence of $n$ increasing random numbers in the interval $[0,1]$ with $\lambda_{0}=0$ and $\lambda_{n}=1.0$. A sequence of $\delta h_{\alpha \beta}^{i}$ specifies the path of a transformation from $I$ to $F$ and satisfies the constraint $\sum_{i=1, \ldots, n} \delta h_{\alpha \beta}^{i}=$ $h_{\alpha \beta}^{F}-h_{\alpha \beta}^{I}$. The work $W$ done along a path is calculated as the sum of the works $w_{i}$ corresponding to each variation $\delta h_{\alpha \beta}^{i}$

$$
W=\sum_{i=1, . ., n} w_{i}=\sum_{i=1, \ldots, n} \int_{V} d \mathbf{r} \sum_{\alpha, \beta} \tau_{\alpha \beta}^{i}(\mathbf{r}) \eta_{\alpha \beta}^{i}
$$

where $\tau_{\alpha \beta}^{i}(\mathbf{r})$ is the tensor of constraint at position $\mathbf{r}$ of the system when the configuration of the $N$ spheres changes volume according to the variation from $h_{\alpha \beta}^{i}$ to $h_{\alpha \beta}^{i+1}$ and $\eta_{\alpha \beta}^{i}$ is the deformation induced by this variation [26]. In the DHS system, the contribution of the dipolar interaction $\tau_{\alpha \beta}^{i, d d}(\mathbf{r})$ is

$$
\tau_{\alpha \beta}^{i, d d}(\mathbf{r})=\frac{1}{2} \sum_{l=1, N} \delta\left(\mathbf{r}-\mathbf{r}_{l}^{i}\right) \sum_{k(\neq l)=1, N} r_{l, \alpha}^{i} F_{l, k, \beta}^{i}
$$

where $F_{l, k, \beta}^{i}$ is the $\beta$ component of the dipole-dipole force acting between the spheres $l$ et $k$. The contribution of the hard core potential is obtained from the expression of the tensor of constaints derived by Allen [27, 28]. The deformation $\eta_{\alpha \beta}^{i}$ corresponds to the change in positions of the spheres entailed by the variation of the volume, i.e. for sphere $l: r_{l, \alpha}^{i+1}-r_{l, \alpha}^{i}=\sum_{\gamma, \beta} h_{\alpha \gamma}^{i+1} \bar{h}_{\gamma \beta}^{i} r_{l, \beta}^{i}-r_{l, \alpha}^{i}$, with $\bar{h}_{\alpha \beta}^{i}$ the inverse matrix of $h_{\alpha \beta}^{i}$. It is identical at each point of the volume and given by

$$
\eta_{\alpha \beta}^{i}=\frac{1}{2}\left(\sum_{\gamma} \delta h_{\alpha \gamma}^{i} \bar{h}_{\gamma \beta}^{i}+\sum_{\gamma} \delta h_{\beta \gamma}^{i} \bar{h}_{\gamma \alpha}^{i}+\sum_{\nu}\left(\sum_{\gamma} \delta h_{\alpha \gamma}^{i} \bar{h}_{\gamma \nu}^{i} \sum_{\gamma} \delta h_{\nu \gamma}^{i} \bar{h}_{\gamma \beta}^{i}\right)\right)
$$

The Jarzynski equality and Jensen's inequality [29] imply the inequality $<W>\geq$ $\Delta F$ where $\langle W\rangle$ is the average $W$ calculated for the ensemble of transformations from $I$ to $F$. The average value, $\left\langle W^{\prime}\right\rangle$, of the work $W^{\prime}$ necessary to make the inverse transformation $F$ to $I$, satisfies the inequality $-<W^{\prime}>\leq \Delta F$. These two 
inequalities giving upper and lower bounds for $\Delta F$, can be used to estimate $\Delta F$ in a solid-solid phase transition [30].

The matrices $h_{\alpha \beta}^{I}$ and $h_{\alpha \beta}^{F}$ specifying the initial and final states of the transformations from the bcc lattice to the bct, bco or fcc lattices are such that if $\mathbf{r}_{1}^{I}, \mathbf{r}_{2}^{I}, \ldots, \mathbf{r}_{N}^{I}$ are the lattice positions of the spheres on the bcc lattice, $\mathbf{r}_{1}^{F}, \mathbf{r}_{2}^{F}, \ldots, \mathbf{r}_{N}^{F}$ obtained from $r_{l, \alpha}^{F}=\sum_{\beta, \gamma} h_{\alpha \gamma}^{F} \bar{h}_{\gamma \beta}^{I} r_{l, \beta}^{I}$, will be those of the spheres on the bct, bco or fcc lattices. In these cases the transformations from $h_{\alpha \beta}^{I}$ to $h_{\alpha \beta}^{F}$ can be made continuously, the volume $V$ maintaining its parallelepipedic shape. The transformation from the bcc to the hcp lattice is also continuous but a relative shift of neighbouring planes, e. g. those perpendicular to the $z$-direction has to be supplemented with the volume change. The initial volume of the bcc lattice is chosen monoclinic and this shape is conserved in the evolution towards the hcp lattice. The lattice positions of the final hcp lattice are related to those of the bcc lattice by $r_{l, \alpha}^{F}=\sum_{\beta, \gamma} h_{\alpha \gamma}^{F} \bar{h}_{\gamma \beta}^{I} r_{l, \beta}^{I}+g \delta_{\alpha 2}$. The displacement $g$ along the $y$ direction generates the succession ...ABAB... of [001] planes characteristic of the hcp lattice. The value of $g$ is alternatively equal to 0 (plane A) or $b / 3$ (plane B) where $b$ is the smallest distance along $y$ between two spheres of a [001] plane of the final hcp lattice [31]. At each stage of the evolution of the volume a small random displacement $\delta g^{i}$ is applied to the atoms in B planes subject to the constraint $\sum_{i=1,2, \ldots, n} \delta g^{i}=b / 3$. These displacements contribute to the values of $w_{i}$ taking into account, in Eq. (3), that the $\eta_{\alpha \beta}^{i}$ differ depending on whether the spheres are located in A or B planes.

The calculation of $W$ along the path from $I$ to $F$ comprises three stages. The first one consists in a simulation in the canonical ensemble to equilibrate the $N$ spheres on the bcc lattice at the chosen values of $\rho^{*}$ et $\mu^{*}$. The second stage starts with the change of the matrix from $h^{0}$ to $h^{1}$. This trial move is either accepted or rejected if there is overlap of the spheres. In the latter case canonical sampling is continued, a new trial transition from 0 to 1 is attempted and the process repeated until acceptance of the transition; $w_{1}$ is then calculated. This procedure is continued until the transition $n-1$ to $n$. The third stage is a simulation in the canonical ensemble at the volume defined by $h_{\alpha \beta}^{n}=h_{\alpha \beta}^{F}$ to calculate the pair correlation functions in the final state $F$. This allows to check that the correlation functions are in agreement with those expected in the bct, bco, fcc or hcp phase for the DHS system at chosen $\rho^{*}$ and $\mu^{*}$.

The random variations $\delta h_{\alpha \beta}^{i}$ are of the order $\left(h_{\alpha \beta}^{F}-h_{\alpha \beta}^{I}\right) / n$. To obtain an acceptance ratio of about $50 \%$ for the transitions $i$ to $i+1, n$ must be of the order of $10^{4}$ at the considered solid phase densities, canonical sampling between transition attempts being $\sim 5 \mathrm{MC}$ trial moves per sphere. The numerical estimate of the hard core contribution to $\tau_{\alpha \beta}(\mathbf{r})$ has been verified for $\mu^{*}=0$ by calculating $\Delta F$ between dense fluid states of hard spheres for which the free energy is known. The Ewald summation method has been applied for the calculation of the energy and $\tau_{\alpha \beta}^{d d}(\mathbf{r})$ [32]. The values of $N$ are 1024 for the calculation of the transitions from bcc to bct or bco, 1200 for those from bcc to hcp and 1372 for those from bcc to fcc.

\section{Results}

In all calculations of $\Delta F$, the initial configuration was a bcc lattice of spheres completely polarized along the $\mathrm{z}$ axis (polarization 1 ). In the density region $1.06 \leq$ $\rho^{*} \leq 1.26$, at $\mu^{*}=1$, the equilibrium state associated with this initial configuration has zero polarization. At $\mu^{*}=2$ and 3 , it has a polarization between 0.75 and 0.87 . These equilibrium states are the reference states used to estimate $\Delta F$ relative to 
the other solid phases. For each chosen thermodynamic state the average value of $e^{-W / k_{B} T}$ has been calculated for 16 paths between the reference bcc state and the final state of symmetry bct, bco, fcc or hcp. Each path is defined by an initial equilibrium bcc configuration and a succession of different random variations $\delta h_{\alpha \beta}^{i}$. This number of different paths turned out to be sufficient because the values of $W$ have a low statistical variance if amplitude and orientation of the polarizations are similar in the final states. The statement has been verified by calculating, at $\mu^{*}=3$ and $\rho^{*}=1.20$, the values of $W$ for 64 paths corresponding to a transition from bcc to bct. To test the method a comparison has been made with results for the phase diagram of the totally polarized hard sphere system $[16,17]$. In agreement with this study, the value of $\Delta F$ obtained from the Jarzynski equality shows that among the phases bct, hcp and hcp compressed along $z$ by about $10 \%$, the phase of bct symmetry is the most stable at $\mu^{*}=2$ and $\rho^{*}=1.20$ and the compressed phase of hcp symmetry the most stable at $\mu^{*}=3$ and $\rho^{*}=1.06$.

In spite of the constraints imposed by the excluded volume effects and the homogeneity of the deformations $\eta_{\alpha \beta}$ it is possible that the transition from the bcc phase to another phase is accompanied by creation of defects. The contribution of these possible defects to $\Delta F$ seems marginal. Indeed, as mentioned above, the variance of the values of $\Delta F$ is small for states with close final polarizations. Moreover, the pair correlation functions calculated in the final states are in excellent agreement with those obtained in canonical simulations at fixed density at identical thermodynamic states. An example of the agreement is given in Fig. 2 for the bcc-bct transition at $\mu^{*}=3$ and $\rho^{*}=1.16$. At given density the bct lattice parameters are obtained from the bcc parameters by multiplying them by $(3 / 2)^{1 / 6},(3 / 2)^{1 / 6}$ and $(2 / 3)^{1 / 3}$ along $x, y$ and $z$. For the bco lattices these factors are $\lambda(3 / 2)^{1 / 6}$, $(3 / 2)^{1 / 6} / \lambda$ and $(2 / 3)^{1 / 3}$ along $x, y$ and $z$ with $\lambda=1.05,1,10,1.15$ and 1.20 in our simulations.

For the states $\mu^{*}=1$ and $\rho^{*} \geq 1.20$ at which the polarizations vanish both in the initial and final states, the difference $\Delta F^{*}=\Delta F / k_{B} T$ is always positive with a statistical error of the order of $0.1 \Delta F^{*}$, indicating that bcc is the stable phase. This result is confirmed by the NpT simulations as shown in Fig. 3a which compares the pair distribution functions (p.d.f.) at $\rho=1.20$ obtained in the isobaric ensemble, starting from an initial bcc lattice, with that for the final state of the NEW method. At densities $\rho^{*} \leq 1.10$ the bcc phase seems also to be the most stable phase but the values of $\Delta F^{*}$ are small $\sim 0.1$ with a statistical error of $\simeq 0.03$. In the domain $1.10 \leq \rho^{*} \leq 1.20$, the bco phase with $\lambda \simeq 1.10$ is marginally more stable than bcc, $\Delta F^{*} \simeq-0.1$. Again the NpT calculation transforms the initial bcc lattice into a state with p.d.f. in good agreement with that of the bco lattice with $L_{y} / L_{x}=1.10$ identified by the NEW method as having the lowest free energy (cf. Fig. 3b).

When $\mu^{*}$ increases the solid phases get polarized. At $\mu^{*}=2$ and increasing densities $1.06 \leq \rho^{*}<1.20$ the most stable phase changes from bct to bco with values of $\lambda$ between 1.05 and 1.15, the polarization being parallel to the $z$ direction. For these bct and bco phases $\Delta F^{*}$ is of the order -1.3 with a statistical error of about $0.02 \Delta F^{*}$. At $\rho^{*} \geq 1.20$, the variance of $\Delta F^{*}$ increases. As a matter of fact, for approximately $15 \%$ of the bco final states, i. e. 2 to 4 out of 16 , the polarization is not along $z$ but either along a diagonal of the parallelepipedic volume or a diagonal of the $x z$ or $y z$ planes and $\Delta F^{*}$ is equal to $\simeq-0.8$. However, the states polarized along $z$ have the lowest free energy $\Delta F^{*} \sim-1.1$ and their equilibrium p.d.f's are in close agreement with those obtained from NpT simulations (cf. Fig. $3 \mathrm{c})$.

If the final states are fcc or hcp, at $\mu^{*}=2, \Delta F^{*}$ varies from $\sim 0.3$ for $\rho^{*}=1.06$ to $\sim-0.5$ for $\rho^{*}=1.20$ then gets positive again $\sim 0.5$ for $\rho^{*}=1.26$. As for the bcc and 


\section{Page $\mathbf{7}$ of $\mathbf{2 1}$

bco phases, the final states are generally polarized along $z$ but other polarization states exist as well where the orientation lies in a lattice plane. Moreover, for the fcc lattice, there exists polarized states, where, as described above in Section II, the average orientation of dipole moments can differ from one domain of lattice planes to the other, while having the same tilt angle with respect to $z$. However the different polarization states of the fcc and hcp phases all have a free energy which is higher by $\sim 1$ compared to the bct or bco phases.

The bct and bco phases with $\lambda<1.15$ have nearly identical stability at $\mu^{*}=3$ and $\rho^{*} \leq 1.20$, differences in $\Delta F^{*}$ being close to the statistical error. The bco phase is marginally more stable at $\rho^{*}=1.26$ with $\lambda \simeq 1.10$. The polarization of the bct or bco phases of lowest free energy is parallel to $z$ and equals $\sim 0.90-0.95$. A typical value of $\Delta F^{*}$ between bcc and bct or bcc and bco is $\sim-4.3$. For the hcp or fcc phases $\Delta F^{*}$ is larger by 1.5 for all polarizations of the final state. Comparison of the p.d.f. at $\mu=3$ for $\rho=1.16$ and $\rho=1.26$ obtained either by NpT simulation or NEW method are shown in Figs. $4 \mathrm{a}$ and $4 \mathrm{~b}$, respectively.

The lattices associated with the most stable phases of the final states have been called, somewhat improperly, with reference to the perfect lattices of hard spheres with volume compatible with the periodic boundary conditions defined by the matrices $h_{\alpha \beta}^{F}$. It is noteworthy that such an appellation is approximate. Upon increase of $\mu^{*}$ the local order of the hard spheres is strongly modified by the dipolar interaction. Figures 5 and 6 are examples of the reorganization of the local order at $\rho^{*}=1.06$ and 1.26. The correlation functions show that if at $\mu^{*}=1$ or 2 the order is close to that of hard spheres $\left(\mu^{*}=0\right)$, it differs significantly at $\mu^{*}=3$. This reorganization is made explicit in Fig. 6 for $\rho^{*}=1.26$. Although the positions of the peaks corresponding to the most numerous neighbours are preserved, peaks of other layers can overlap with these main peaks, or overlap between themselves; occasionally new peaks can appear. These rearrangements result from the open character of the local order in the bct and bco lattices; they are less important in the compact lattices like fcc where they vanish for densities $\rho^{*} \geq 1.20$ (cf. Figs. 7 and 8). The partial delocalization of shells of neighbours thus does not allow a complete characterization of the cristalline order from the sole pair correlation functions. This is particularly clear for perfect bco lattices in which the number of nearest neighbors in successive shells are $2,8,2,2,4,4,8,4, \ldots$, respectively. At densities $\rho^{*} \sim 1.20$, the locations of the first and second shells and those of the seventh and eighth shells differ only by $\sim 0.002 \sigma$ so that these shells merge due to the molecular motion.

When the final states are divided into states of different polarizations they will have different local hard sphere order. These differences in structural arrangement explain the difference in free energies of these final states. Thus, at $\mu^{*}=3.0$ and $\rho^{*}=1.26$, the final states bct and bco, with polarization parallel to $z$ have $\Delta F^{*} \sim-4.0$ whereas those with polarization along a diagonal of the volume have a higher free energy $\Delta F^{*} \sim-1.0$. Fig. 9 shows the change of local order entailed by these variations of free energy and polarization.

In the case where the probability distribution $W$ is gaussian of width $\sigma_{W}$, it is easy to show from the Jarzynski equality that [20]

$$
\Delta F=<W>-\frac{\sigma_{W}^{2}}{2 k_{B} T}
$$

Our results are compatible with this equality provided the polarizations of the final states are unique. When several states of polarization are possible, the distribution of values $W$ has several peaks, is not gaussian and the equality (6) is not valid 
anymore.

\section{Discussion}

The results for the free energy differences calculated from Eq.(1) are summarized in Fig. 10 which shows the domains of stability of the bcc, bct and bco phases with an approximate location of their limits as a function of $\mu^{*}$ and $\rho^{*}$. Only these phases are plotted since the NEW method results, as dicussed in Sect. 3, indicate that for this domain of $\mu^{*}$ and $\rho^{*}$ values, the fcc and hcp phases are always unstable. They are in relative agreement with those given by the simulations in the isobaric ensemble. The solid phases identified by the NEW method as having the lowest free energy (among those considered) and those obtained by the simulations in the NpT ensemble are in good agreement for solid densities $\rho^{*} \geq 1$.16. In particular the local order obtained from the p.d.f. are quasi identical. Additional NpT calculations in this density region at $\mu^{*}=1.5$ and $\mu^{*}=2.5$ also predict structures compatible with those obtained by the NEW method simulations at $\mu^{*}=2$ and $\mu^{*}=3$. At the lower densities $1.06 \leq \rho^{*} \leq$ 1.10 such an agreement could not be found. For $\mu^{*}=2$ or 3 , although the free energy calculations show that the bct phases or the bco phases with ratio $L_{y} / L_{x}$ close to 1.0 are the most stable, the NpT simulations do not evolve spontaneously towards solid arrangements of this type. With different initial choices of the initial configuration the NpT simulations stabilize in a variety of states manifesting the possible existence of several metastable states close to the state of minimum free energy. By comparison of the pair distribution functions, it appears that these states obtained by the NpT simulations do not belong to the set of final states considered in the NEW computations; therefore the relative values of their free energy could not be estimated.

\section{Conclusion}

Use of the Jarzynski equality provides an alternative to the method of thermodynamic integration for the estimate of free energy differences between equilibrium thermodynamic states. The reliability of the calculation relies on the choice of the paths selected to calculate the average in Eq. (1) [33-35]. As mentioned above, by associating to the variation of $h_{\alpha, \beta}$ the relaxation of the sphere configurations, it is possible to prevent overlap of the hard cores to interrupt the transition from one phase to the other. Moreover, if by construction of the transition path one has $V^{F}=V^{I}$, the volumes $V^{i}$ differ from $V^{F}$ only by $0.05 \%$ thus avoiding creation of defects. The choice of the paths seems justified by the facts that Eq. (6) is satisfied and that, taking into account the relaxation of the intermediate configurations and the number of values of $n$ of order 10000 to 30000, the paths nearly satisfy the equality valid only for reversible equilibrium paths

$$
F_{F}-F_{I}=\sum_{i=1, n} \int d \mathbf{r} \sum_{\alpha, \beta}<\tau_{\alpha \beta}^{i}(\mathbf{r})>_{i} \eta_{\alpha, \beta}^{i}(\mathbf{r}),
$$

where $\langle\ldots\rangle_{i}$ denotes a canonical average at step $i$ of a transition path.

As stated in the introduction the crossing through a first order phase transition precludes that Eq. (7) can be a strict equality, contrary to the Jarzynski equality. This illustrates again the relative advantages of the 
NEW and thermodynamic integration methods. Eq. (1) is always valid and needs only an initial reference state. It requires though to perform an average on a set of adequately chosen paths and, in the present work, the rather time comsuming computation of the hard sphere tensor of constraint. The thermodynamic integration method is simple to implement and uses only one path. However, to circumvent the first order phase transition problem, especially for solid phases, it needs to use multiple initial reference states the free energy of which must be known analytically. Such initial states, well adapted to hard sphere systems with rotational degrees of freedom, seem difficult to construct.

In conclusion, the solid phases of dipolar hard spheres estimated by free energy calculations to be the most stable ones are of the bcc, bct or bco type. For dipole moments $\mu^{*} \geq 2$ these phases are polarized. For the bct and bco phases the direction of polarization is the direction along which the bcc unit cell is compressed (in our calculations the $\mathrm{z}$ direction). The pair distribution functions show, however, that the local arrangements of a particle and its nearest neighbours can differ notably according to the values of $\rho^{*}$ and $\mu^{*}$. A detailed and complete characterization of the unit cell of these phases remains difficult, in particular, due to the proximity of nearest neighbour shells and the small number of particles in these shells (cf. Sect. 3).

The NpT simulation results are in accord with the free energy results for thermodynamic states of fixed $\mu^{*}$ and densities larger than roughly 1.10 and confirm in this domain the phase diagram given in Fig. 10. For the states $\mu^{*} \geq 2$ and densities lower than approximately 1.10 , although the stable phases are identified without ambiguity from the free energy calculations, this domain of the phase diagram has to be confirmed by a method different from that based on the NEW method.

\section{References}

[1] J.-J. Weis and D. Levesque, Adv. Polym. Sci. 185, 163 (2005).

[2] D. Wei and G. Patey, Phys. Rev. Lett. 76, 3348 (1992).

[3] J.-J Weis, D. Levesque and G. Zarragoicoechea, Phys. Rev. Lett. 69, 913 (1992).

[4] M. Stevens and G. Grest, Phys. Rev. E 51, 5962 (1995).

[5]P.J. Camp, J.C. Shelley and G.N. Patey, Phys. Rev. Lett. 84, 115 (1999).

[6] G. Ganzenmüller and P.J. Camp, J. Chem. Phys. 126, 191104 (2007).

[7]R. Jia, H. Braun, and R. Hentschke, Phys. Rev. E 82, 062501 (2010).

[8]D. Wei and G. Patey, Phys. A 46, 7783 (1992).

[9] J.-J Weis, J. Chem. Phys. 123, 044503 (2005).

[10] J.-J Weis and D. Levesque, J. Chem. Phys. 125, 034504 (2006).

[11] K.I. Morozov, J. Chem. Phys. 119, 13024 (2003).

[12] S.T. Lagerwall, J. Phys.: Condens. Matter 8, 9143 (1996).

[13] J.-J Weis and D. Levesque, Phys. Rev. E 48, 3728 (1993).

[14]B. Groh and S. Dietrich, Phys. Rev. E 63, 021203 (2001).

[15] G.T. Gao and X.C. Zeng, Phys. Rev. E 61, R2188 (2000).

[16] A. P. Hynninen and M. Dijkstra, Phys. Rev. Lett. 94, 138303 (2005).

[17] A. P. Hynninen and M. Dijkstra, Phys. Rev. E 72, 051402 (2005).

[18] A.B. Belonoshko, O. LeBacq, R. Ahuja and B. Johansson, J. Chem. Phys. 117, 7233 (2002).

[19] D. Frenkel and B. Smit, Understanding Molecular Simulation (Academic, San Diego, 1996).

[20]C. Jarzynski, Phys. Rev. Lett. 97, 2690 (1997).

[21]M.P. Allen and D.J. Tildesley, Computer Simulation of Liquids (Clarendon, Oxford, 1989).

[22] C. Jarzynski, Phys. Rev. E 56, 5018 (1997).

[23]E. Schöll-Paschinger and G. Dellago, J. Chem. Phys. 125, 054105 (2006).

[24] G. E. Crooks, Phys. Rev. E 60, 2721 (1999).

[25] G. E. Crooks, Phys. Rev. E 61, 2361 (2000).

[26]S. Marante, G.C. Rossi and M. Testa, J. Chem. Phys. 125, 034101 (2006).

[27] M. Allen, J. Chem. Phys. 124, 214103 (2006).

[28] A. Trokhymchuk and J. Alejandre, J. Chem. Phys. 111, 8510 (1999).

[29]D. Chandler, Introduction to Modern Statistical Mechanics (Oxford, New York, 1987).

[30] R.S. Hoy and M.O. Robbins, Phys. Rev. E 69, 056103 (2004).

[31] K.J. Caspernen and E.A. Carter, PNAS, 102, 6738, (2005).

[32] A. Aguado and P.A. Madden, J. Chem. Phys. 119, 7471 (2003).

[33] C. Jarzynski, Phys. Rev. E 65, 046122 (2002). 
[34]F. M. Ytreberg, R. H. Swendsen, and D. M. Zuckerman, J. Chem. Phys. 125, 184114 (2006).

[35]S. Vaikuntanathan and C. Jarzynski, J. Chem. Phys. 134, 054107 (2011). 


\section{Figure Captions}

Fig. 1: Projection on the $x y$ plane of the dipole moments for a fcc lattice with polarization along the $x$ direction at $\rho^{*}=1.26$ and $\mu^{*}=3.0$ (NVT simulation with $\mathrm{N}=1372$ ).

Fig. 2: Comparison of the pair distribution function $g(r)$ in the final state of the transition from the bcc phase to the bct phase (black line) with that obtained from a canonical ensemble calculation in the bct phase (red line). The density is $\rho^{*}=1.16$ and the dipole moment $\mu^{*}=3$.

Fig. 3: Comparison of equilibrium pair distribution functions of the lowest free energy states obtained from NEW method (dashed line and dots) and pair distribution functions of stable final states resulting from NpT simulation (solid line) a) $\mu^{*}=1$ and $\rho^{*}=1.20$, the lowest free energy state is bcc; the NpT simulations are started from an initial bcc lattice; b) $\mu^{*}=1$ and $\rho^{*}=1.10$, the lowest free energy state is bco with $L_{y} / L_{x}=1.10$; the NpT simulations are started from an initial bcc lattice; c) $\mu^{*}=2$ and $\rho^{*}=1.20$, the lowest free energy state is bco with $L_{y} / L_{x}=1.05$; the NpT simulations start from an initial bct lattice.

Fig. 4: Comparison of equilibrium pair distribution functions of the lowest free energy states obtained from NEW method (dashed line and dots) and pair distribution functions of stable final states resulting from $\mathrm{NpT}$ simulation (solid line) a) $\mu^{*}=3$ and $\rho^{*}=1.16$, the lowest free energy state is bct; the NpT simulations are started from an initial bct lattice; b) $\mu^{*}=3$ and $\rho^{*}=1.26$, the lowest free energy state is bct; the NpT simulations are started from an initial bct lattice.

Fig. 5: Pair distribution function $g(r)$ for equilibrium states of a bct phase at $\rho^{*}=1.06$ and increasing values of $\mu^{*}$. For $\mu^{*}=2$ and 3 the polarizations are parallel to $z$ and equal to $\sim 0.9$.

Fig. 6: Pair distribution functions $g(r)$ for equilibrium states of a bct phase at $\rho^{*}=1.26$ and increasing values of $\mu^{*}$ values. For $\mu^{*}=2$ and 3 the polarizations are parallel to $z$ and equal to $\sim 0.9$.

Fig. 7: Pair distribution functions $g(r)$ for equilibrium states of a fcc phase at $\rho^{*}=1.06$ and increasing values of $\mu^{*}$. For $\mu^{*}=2$ and 3 the polarizations are parallel to $z$ and equal to $\sim 0.75$.

Fig. 8: Pair distribution function $g(r)$ for equilibrium states of a fcc phase at $\rho^{*}=1.26$ and increasing values of $\mu^{*}$. For $\mu^{*}=2$ and 3 the polarizations are parallel to $z$ and equal to $\sim 0.75$.

Fig. 9: Pair distribution functions $g(r)$ for final equilibrium states of a bcc to bct phase transition corresponding to different orientations of the polarization at $\rho^{*}=$ 1.26 and $\mu^{*}=3.0$. Solid line: polarization parallel to $z$ axis; red line: polarization parallel to a diagonal of the parallelepipedic volume.

Fig. 10: Phase diagram obtained from free energy differences computed by the Jarzynski equality (NEW method). Two superimposed symbols for a state indicates close stability of the two phases. 


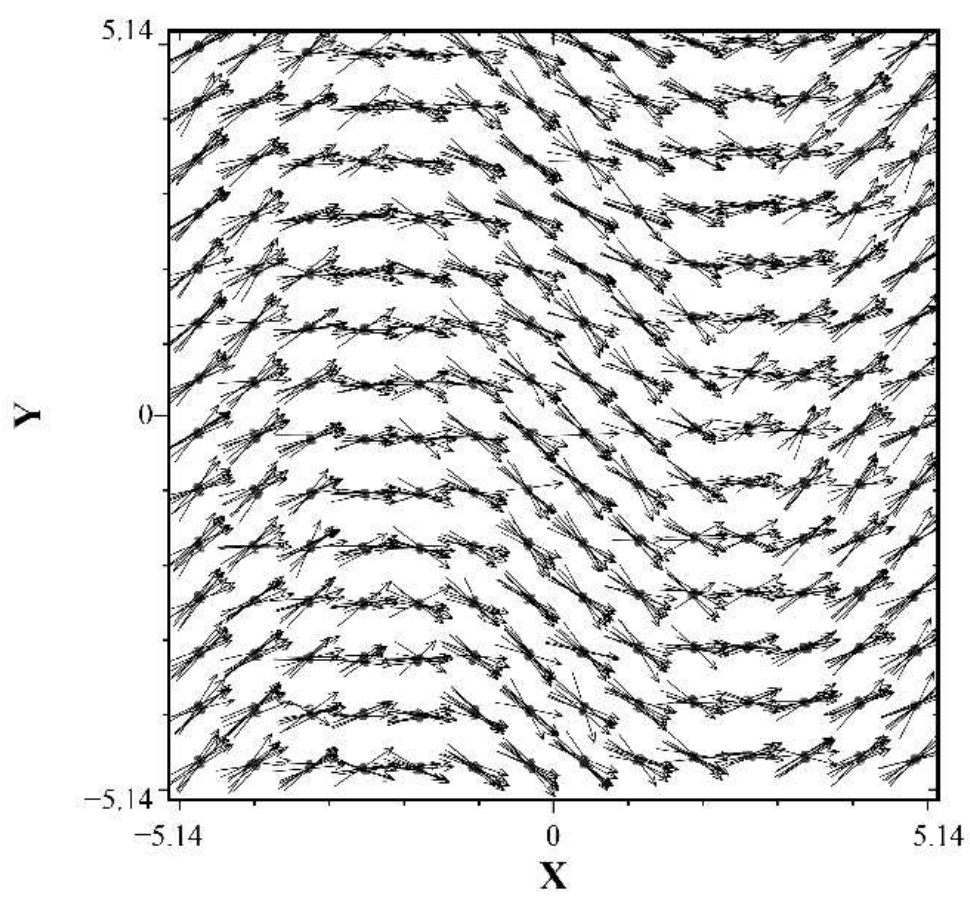

Fig. 1

URL: http://mc.manuscriptcentral.com/tandf/tmph 
1

2

3

4

5

6

7

8

10

11

12

13

14

15

16

17

18

19

20

21

22

23

24

25

26

27

28

29

30

32

34

35

36

37

38

39

40

41

42

43

44

45

46

47

48

49

50

51

52

53

54

55

56

57

58

59

60

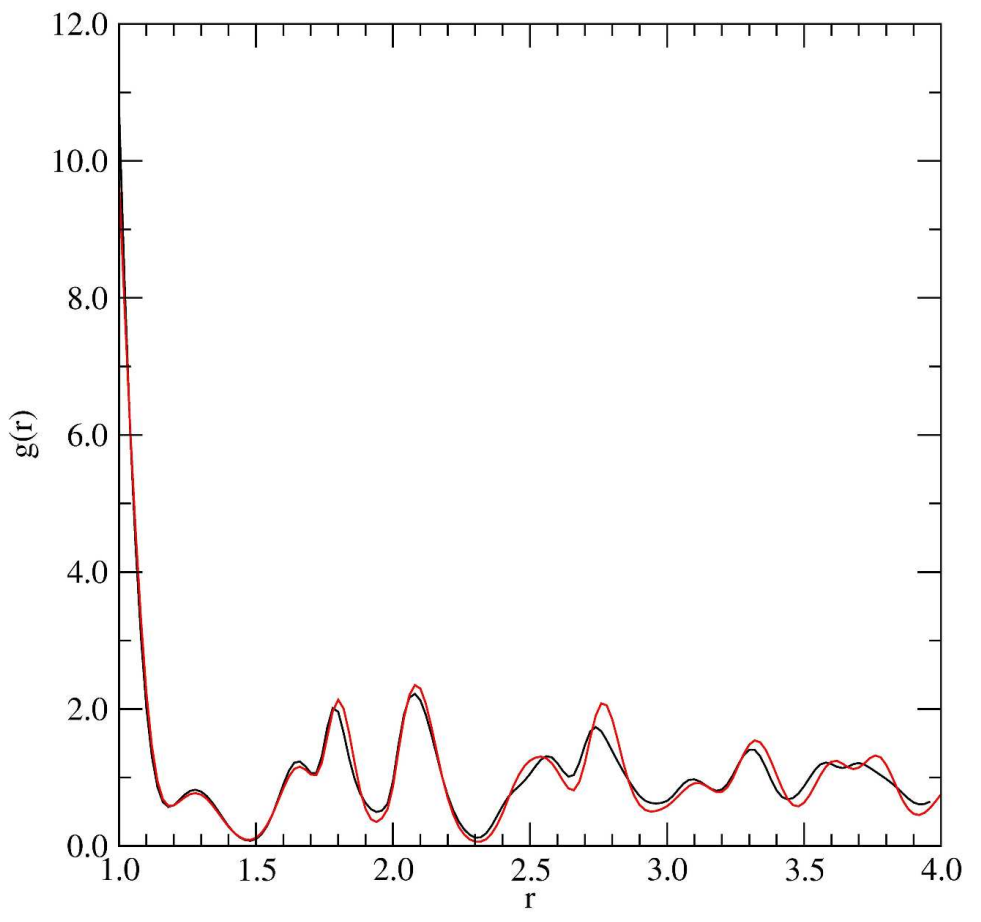

Fig. 2

$279 \times 361 \mathrm{~mm}(300 \times 300$ DPI $)$

URL: http://mc.manuscriptcentral.com/tandf/tmph 

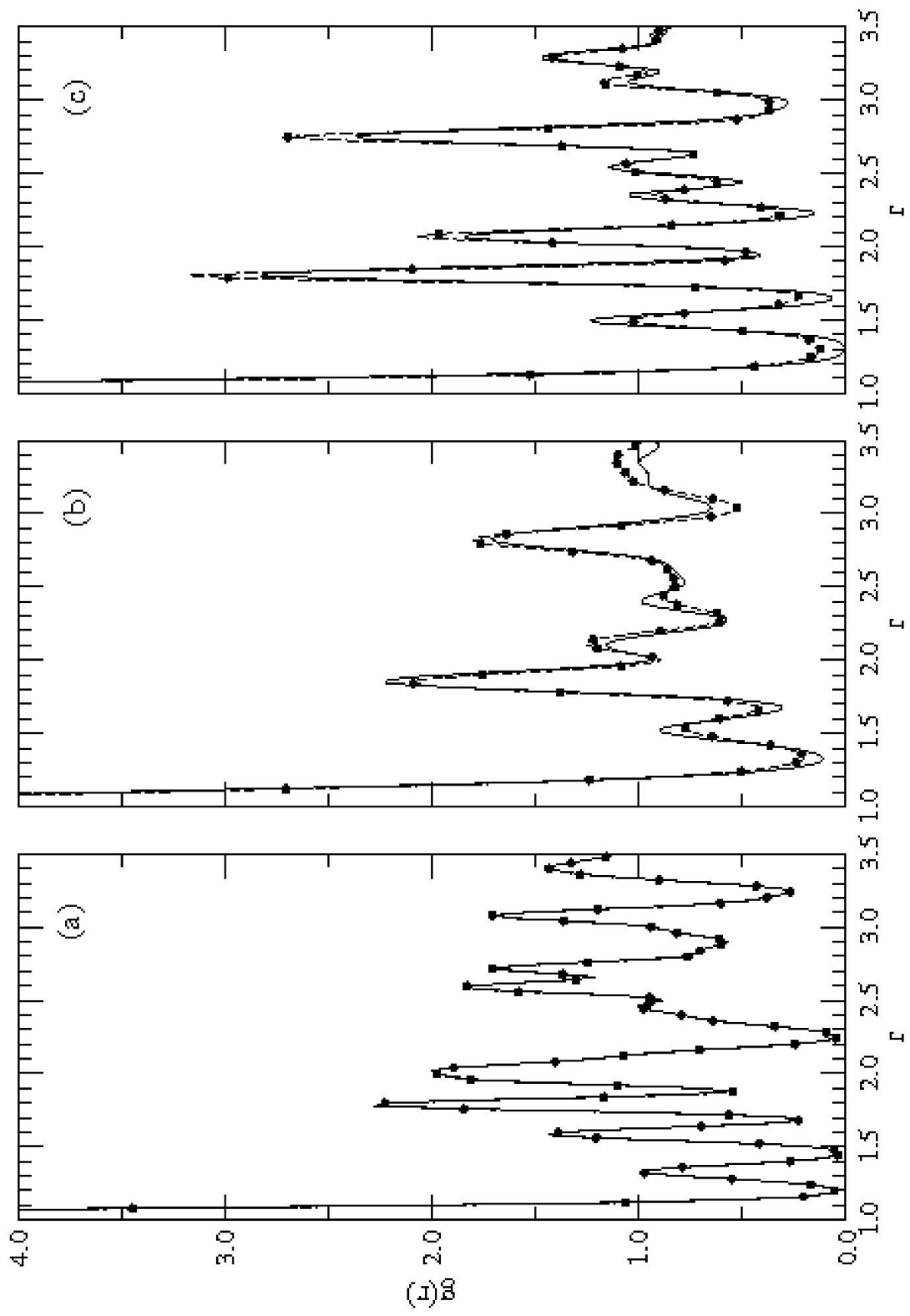

Fig. 3

$231 \times 335 \mathrm{~mm}(300 \times 300 \mathrm{DPI})$

URL: http://mc.manuscriptcentral.com/tandf/tmph 

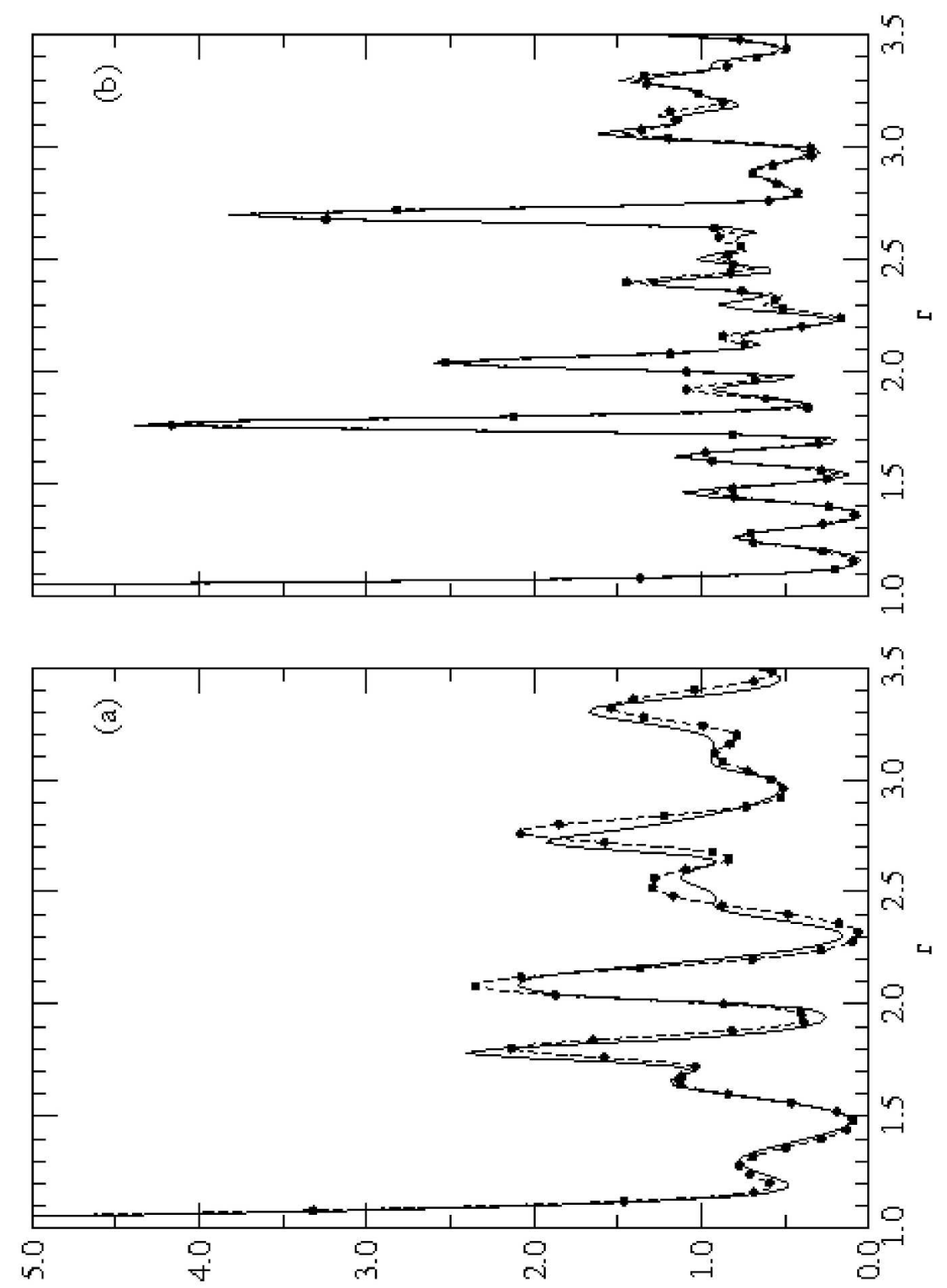

(I) 8

Fig. 4

$231 \times 331 \mathrm{~mm}(300 \times 300$ DPI $)$

URL: http://mc.manuscriptcentral.com/tandf/tmph 


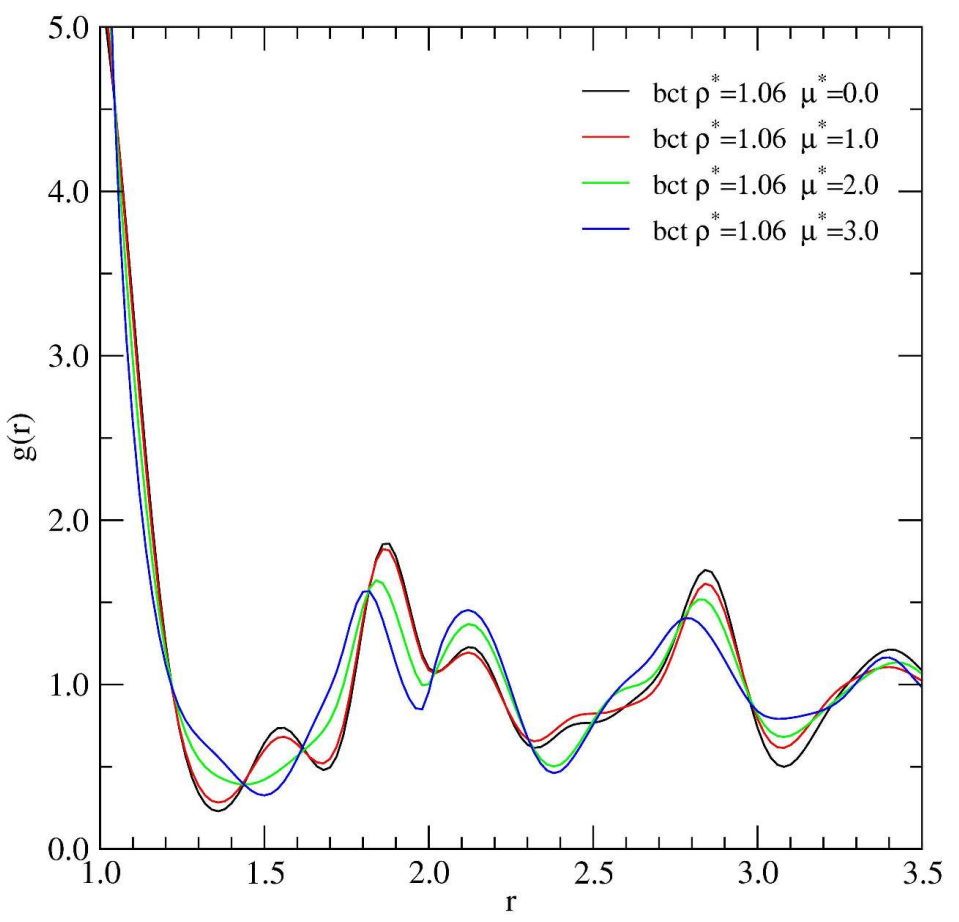

Fig. 5

$279 \times 361 \mathrm{~mm}(300 \times 300 \mathrm{DPI})$

URL: http://mc.manuscriptcentral.com/tandf/tmph 


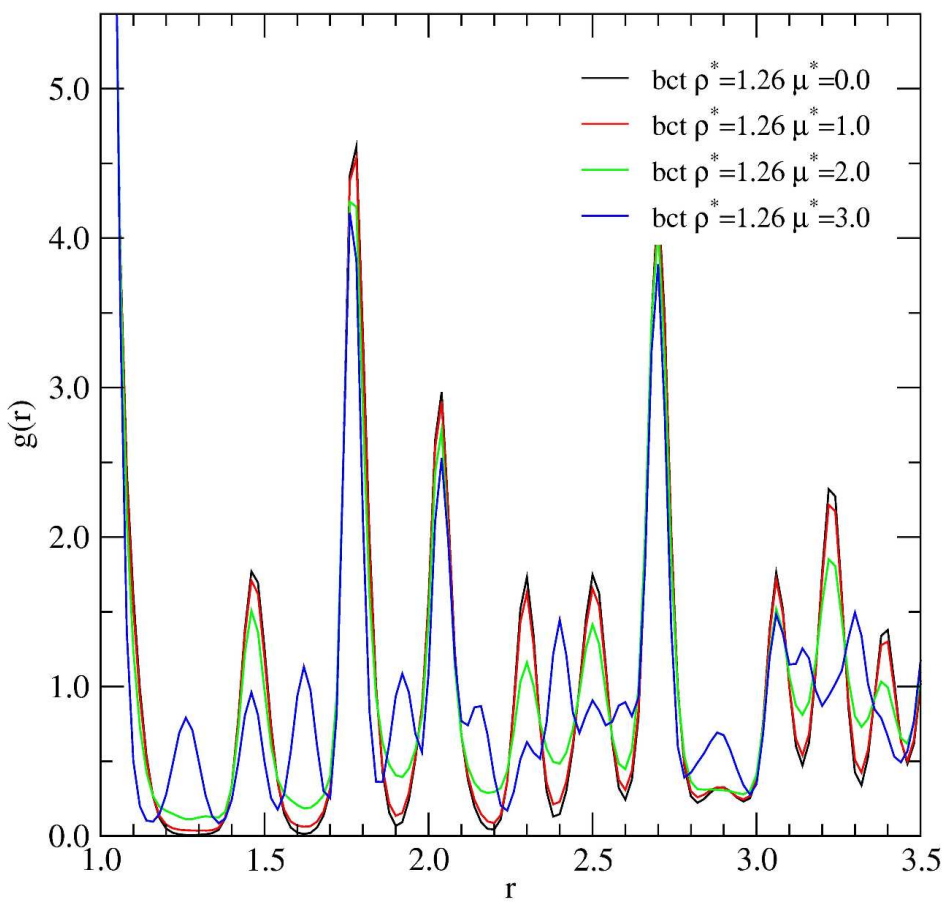

Fig. 6 $279 \times 361 \mathrm{~mm}(300 \times 300 \mathrm{DPI})$ 


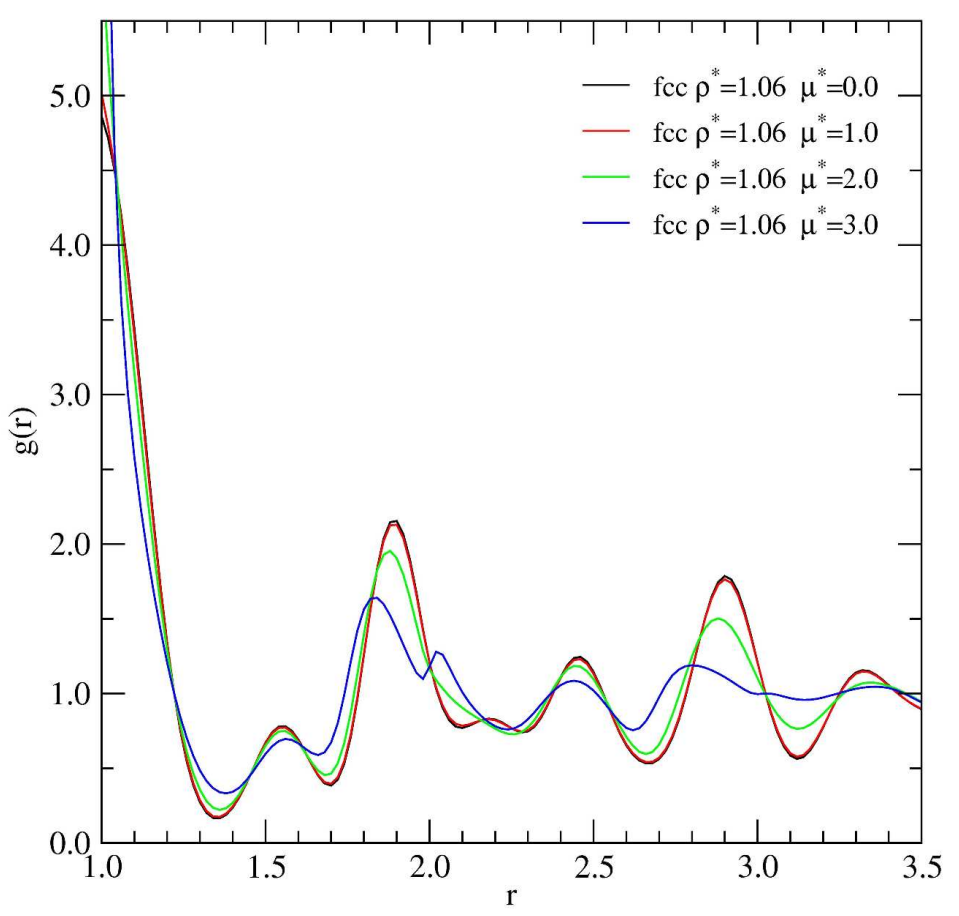

Fig. 7

$279 \times 361 \mathrm{~mm}(300 \times 300 \mathrm{DPI})$

URL: http://mc.manuscriptcentral.com/tandf/tmph 


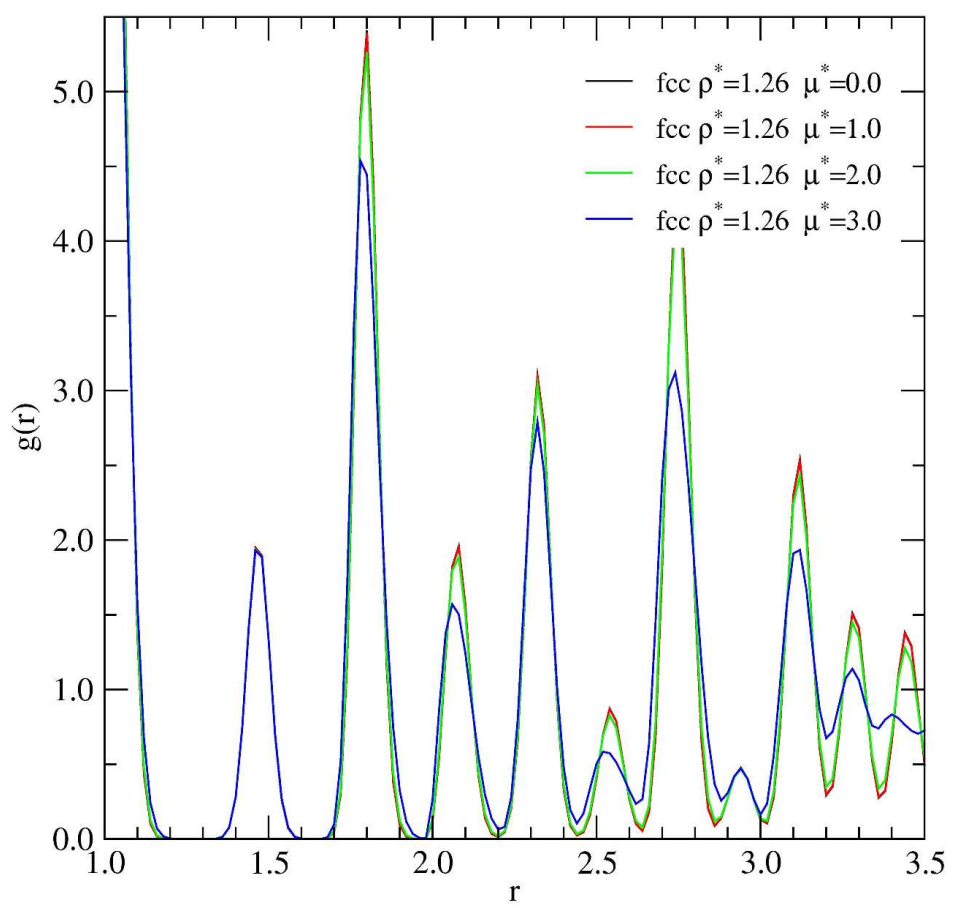

Fig. 8 $279 \times 361 \mathrm{~mm}(300 \times 300 \mathrm{DPI})$ 


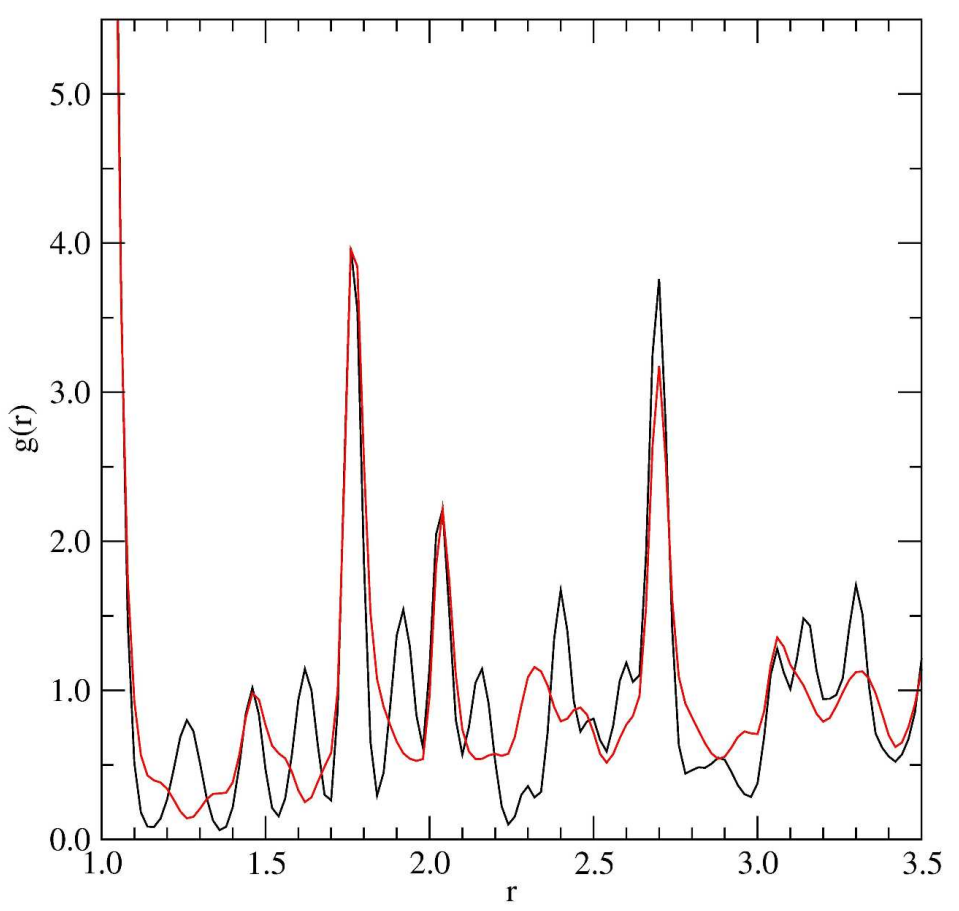

Fig. 9

$279 \times 361 \mathrm{~mm}(300 \times 300$ DPI $)$

URL: http://mc.manuscriptcentral.com/tandf/tmph 


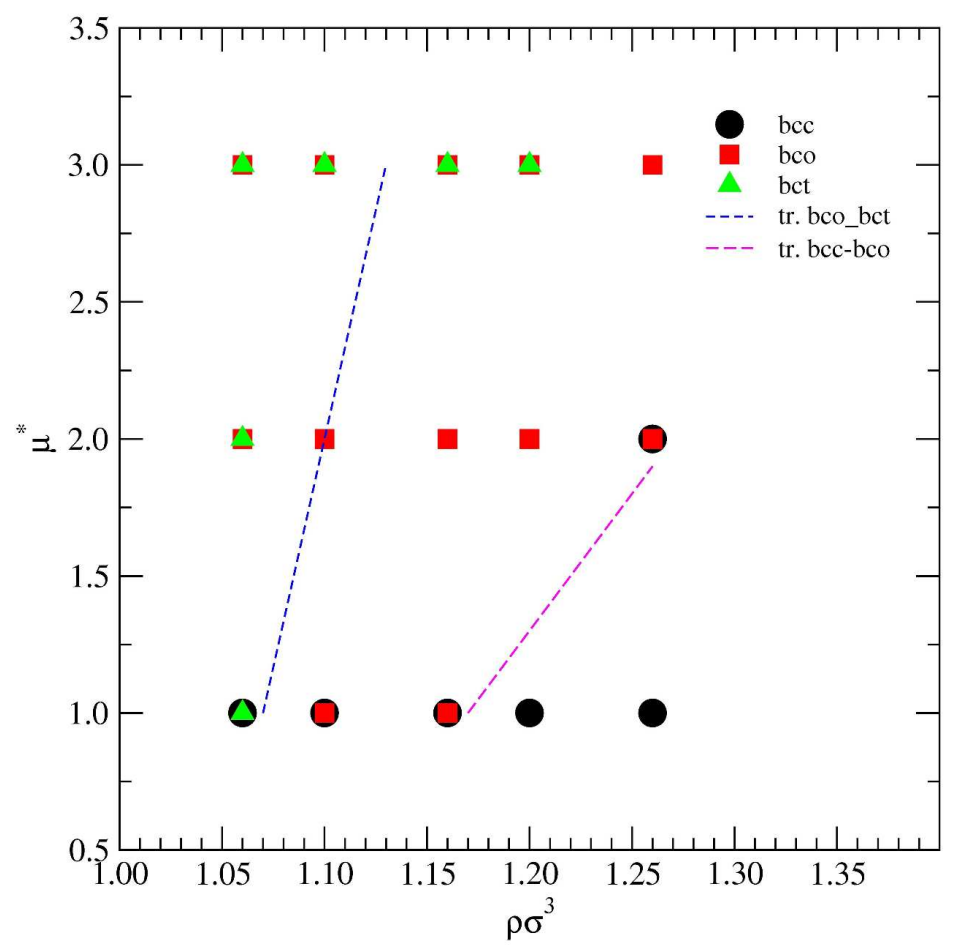

Fig. 10

$279 \times 361 \mathrm{~mm}(300 \times 300 \mathrm{DPI})$

URL: http://mc.manuscriptcentral.com/tandf/tmph 